\title{
Hungry for knowledge-the role of quality improvement projects in Canadian anesthesia departments
}

\author{
Kathryn Anne Sparrow, MD, FRCPC, MScHQ®
}

Received: 3 February 2020/Revised: 3 February 2020/Accepted: 19 February 2020/Published online: 9 March 2020

(c) Canadian Anesthesiologists' Society 2020

In this issue of the Journal, Merchant presents the findings of a retrospective quality review that collected baseline data on elective operating room $(\mathrm{OR})$ scheduled start time adherence to inform institutional perioperative patient fasting instructions at the Fraser Health Authority (FHA) in British Columbia, Canada. ${ }^{1}$

Review of slate scheduling information from 123,865 elective non-cardiac cases in 11 FHA hospitals over a 32month period showed that it was uncommon for patients to enter the OR more than 90 min early ( $0.6 \%$ of all cases). Establishing baseline OR schedule adherence data makes the OR schedule a reliable guide to providing fasting instructions in appropriately selected elective FHA surgical patients that are consistent with the Canadian Anesthesiologists' Society's (CAS) Guidelines to the Practice of Anesthesia. ${ }^{2}$

Aspiration of gastric contents is still an important cause of morbidity and mortality associated with airway management, and was the most common cause of death in anesthesia cases reported to the 4th National Audit Project. ${ }^{3}$ Sequelae range in severity from mild symptoms, such as transient hypoxemia, to respiratory failure, acute respiratory distress syndrome, cardiovascular collapse, anoxic brain injury, and death. Although emerging technologies such as point-of care ultrasound ${ }^{4}$ may help determine aspiration risk, preoperative fasting instructions for most Canadian elective patients are guided by the CAS Guidelines. ${ }^{2}$ Despite guideline updates and Enhanced Recovery After Surgery programs that encourage clear

K. A. Sparrow, MD, FRCPC, MScHQ ( $\square)$

Discipline of Anesthesia, Memorial University of

Newfoundland, 230 Elizabeth Ave, St. John's, NL A1C 5S7,

Canada

e-mail: ksparrow@mun.ca fluid consumption up to two hours prior to a scheduled procedure, many patients are given instructions for prolonged fasting to maintain scheduling flexibility. ${ }^{5}$

Appropriate implementation of fasting guidelines can minimize adverse patient events (e.g., pulmonary aspiration of gastric contents), minimize intravascular volume depletion, maximize efficiency, minimize case cancellations or delays, and increase patient satisfaction.

The Canadian Anesthesia Research Priority Setting Partnership recently published priorities for patientoriented research and patient engagement. ${ }^{6}$ Scholarly healthcare improvement can help answer many of the top identified priorities to improve outcomes and satisfaction for patients receiving anesthesia for surgery. Scholarly reporting on healthcare improvement is essential to improve the quality, safety, and value of "healthcare for patients, professionals, and the public."7

The findings of Merchant's report will be of interest to all anesthesia providers, as inefficient patient flow may cause delays, increase cost, and reduce patient and staff satisfaction. Optimizing OR flow is important for patient safety and high-quality patient-centred care. ${ }^{8}$ The data from this review can be used to inform future quality improvement (QI) projects and establish a plan for a continuous model of improvement.

Given the increased focus on healthcare quality and culture of safety, recommendations for the integration of healthcare improvement in clinical care, reporting of robust methodological projects, and utilization of incident reporting systems (IRS) will be further explored in this editorial. 


\section{The science of healthcare improvement}

Batalden and Davidoff defined QI as the "combined and unceasing efforts to make the changes that will lead to better patient outcomes (health), better system performance (care), and better professional development (learning)." 9 This definition requires the belief that "everyone in healthcare must recognize that they have two jobs when they come to work every day: doing the work and improving it." 9

As the systemic effort to improve healthcare quality, safety, and value has grown, so too has the science of healthcare improvement. The revised 2015 Standards for Quality Improvement Reporting Excellence (SQUIRE 2.0) emphasizes three key components for scholarly reporting of healthcare improvement: formal and informal theory in planning, implementing, and evaluating improvement work; the context in which work is done; and the study of the intervention. ${ }^{7}$ Efforts to improve healthcare can be complex and context-dependent, and the SQUIRE 2.0 Guidelines were designed to be applied across many methodologic approaches, such as Plan-Do-Study-Act cycles in single settings to multi-site randomized trials. The SQUIRE 2.0 Guidelines ensure consistency in reporting, as improvement draws on the epistemology of a variety of fields.

\section{Integration of QI and patient safety education with clinical care}

Recent CAS Guidelines recommend employing "a systematic approach for monitoring the quality of anesthetic care provided by members of the department of anesthesia throughout the healthcare facility". ${ }^{2}$ A narrative description by Pysyk et al. highlights the creation, management, and experiences of a quality and patient safety (QPS) committee in a Canadian department of anesthesiology and pain medicine. ${ }^{10}$ Although local QPS committee structure and function will be in keeping with local institutional policies and provincial legislation, the authors illustrate the utilization of a robust incident reporting system and institutional clinical care outcome data to institute changes and educate peers on emerging patient safety and quality of care issues.

Quality improvement and patient safety are viewed as core competencies for health professionals such as anesthesiologists and anesthesiology trainees. The Royal College of Physicians and Surgeons of Canada has proposed four strategic directions to integrate QI and patient safety education (QIPS) with clinical care to improve outcomes for patients, their families, and communities: prioritize the integration of QIPS education and clinical care, build structures and implement processes to integrate QIPS education and clinical care, build capacity for QIPS education at multiple levels, and align educational and patient outcomes to improve quality and patient safety. ${ }^{11}$

Although physician engagement is important for project success, anesthesiologists often lack training in QI, which can make participation in healthcare improvement projects challenging. Quality and patient safety committees and IRS, such as the Canadian Anesthesia Incident Reporting System (CAIRS), ${ }^{\mathrm{A}}$ can help identify potential improvement opportunities within departments of anesthesiology, perioperative medicine, and pain management. Silver et al. have published an article that can help familiarize healthcare professionals on designing an healthcare improvement project. ${ }^{12}$ Enlisting a core change team with expertise in the quality of care problem, leadership, and change management will help to identify key stakeholders, including patients. An improvement model, such as Six Sigma, Lean, and the Model for Improvement serves as the scientific framework to guide the process of healthcare improvement. Quality improvement initiatives have been shown to have positive impacts on patient safety and patient care. ${ }^{13}$ Leadership support, data utilization, and internal champions have been identified as important QI facilitators. Barriers to QI success include time availability and prioritization of QI with other duties.

\section{Creating a culture of safety}

Merchant's article highlights the potential limitations of utilizing available information management systems (IMS) for healthcare improvement initiatives. While the FHA operating room database was used to examine case start time adherence, patient safety incidents associated with non-adherence could not be linked to fasting guidelines or scheduling changes to allow for adherence to fasting guidelines using the available IMS. The IMS should be designed to provide searchable data and be used in communication with other facility databases when possible. $^{2}$

Anesthesiologists and other healthcare providers share a common goal of recognizing patient safety incidents, identifying causes, and making system-wide changes to reduce medical risk. The objective of the CAIRS is to collect all incidents, errors, and complications during the perioperative period. ${ }^{14}$ There is a strong belief that perioperative outcomes can be improved by studying and

\footnotetext{
A Canadian Anesthetic Incident Reporting System. Available from URL: www.cairs.ca (accessed February 2020).
} 
understanding the contributors to patient safety incidents at both the local and national level. An integrated IRS framework for the management of safety, quality, and risk, such as CAIRS, is needed.

\section{Conclusions}

Data collection alone cannot effect change. ${ }^{15}$ Successful translation of improvement measures at a local and national level will result in a continuous quality cycle and contribute to the improvement of perioperative patient safety. This will only be possible through a commitment to a "culture of safety" and a Canadian perioperative care system that places excellence in patient care and safety as a top priority.

Dans ce numéro du Journal, Merchant présente les résultats d'un examen rétrospectif de la qualité qu'il a réalisé en colligeant les données de base concernant l'observance de l'heure de début planifiée au programme opératoire des interventions non urgentes afin de guider les directives institutionnelles de jeûne périopératoire pour les patients dans le réseau de l'Autorité sanitaire Fraser (FHA) en Colombie-Britannique, au Canada. ${ }^{1}$

En passant en revue les informations de planification de salle d'opération (SOP) pour 123865 cas non cardiaques non urgents dans 11 hôpitaux FHA sur une période de 32 mois, Merchant a démontré qu'il était rare que les patients entrent en SOP plus de 90 minutes plus tôt que prévu ( $0,6 \%$ de tous les cas). Grâce à l'identification des données de base d'observance du programme opératoire, le programme opératoire devient un guide fiable pour fournir des recommandations de jeûne pour les patients de chirurgie non urgente adéquatement sélectionnés dans le FHA - recommandations qui s'alignent donc avec le Guide d'exercice de l'anesthésie de la Société canadienne des anesthésiologistes (SCA). ${ }^{2}$

L'aspiration du contenu gastrique constitue encore une cause importante de morbidité et de mortalité associée à la prise en charge des voies aériennes, et elle constituait la cause la plus fréquente de décès liée à l'anesthésie rapportés lors du $4^{\mathrm{e}}$ Projet d'audit national britannique. ${ }^{3}$ Les séquelles vont de légers symptômes tels qu'une hypoxémie transitoire à une insuffisance respiratoire, à un syndrome de détresse respiratoire aiguë, à une défaillance cardiovasculaire, à des lésions cérébrales anoxiques jusqu'au décès. Bien que des technologies émergentes telles que l'échographie gastrique ${ }^{4}$ pourraient nous aider à déterminer le risque d'aspiration, les directives de jeûne préopératoire fournies à la plupart des patients canadiens de chirurgie non urgente s'appuient sur le Guide de la $\mathrm{SCA}^{2}$ Malgré les mises à jour des directives et les programmes de Récupération rapide après la chirurgie qui encouragent la consommation de liquides clairs jusqu'à deux heures avant une intervention planifiée, de nombreux patients se font demander de prolonger le jeûne afin de maintenir une flexibilité dans la programmation. ${ }^{5}$

Une bonne mise en œuvre des directives de jeûne peut minimiser les complications pour les patients (par ex., l'aspiration pulmonaire du contenu gastrique), minimiser la déplétion plasmatique intravasculaire, maximiser l'efficacité, minimiser les annulations de cas ou les retards, et augmenter la satisfaction des patients.

Le Partenariat pour l'établissement des priorités pour la recherche en anesthésie au Canada (CAR-PSP - Canadian Anesthesia Research Priority Setting Partnership) a récemment publié une liste des priorités pour la recherche axée sur le patient et l'implication des patients. ${ }^{6}$ L'amélioration érudite des soins de santé peut aider à répondre à bon nombre des priorités identifiées comme plus urgentes pour améliorer les devenirs et la satisfaction des patients recevant une anesthésie pour la chirurgie. La communication érudite à propos des améliorations des soins de santé est essentielle si l'on souhaite améliorer la qualité, la sécurité et la valeur des « soins de santé pour les patients, les professionnels et le public ». ${ }^{7}$

Les résultats du compte-rendu de Merchant sont d'intérêt pour tous les fournisseurs de soins anesthésiques : en effet, un roulement inefficace de patients pourrait provoquer des retards, augmenter les coûts et réduire la satisfaction tant des patients que du personnel. L'optimisation du roulement de la SOP est importante pour la sécurité des patients et pour promouvoir des soins axés sur le patient de qualité élevée. ${ }^{8}$ Les données de ce compte rendu peuvent être utilisées pour guider des projets futurs d'amélioration de la qualité et établir un plan pour un modèle constant d'améliorations.

Étant donné l'attention croissante portée à la qualité des soins de santé et à la culture de la sécurité, cet éditorial s'attardera également sur certaines recommandations pour l'intégration des améliorations des soins de santé dans les soins cliniques, la présentation de projets méthodologiques rigoureux et l'utilisation des systèmes de notification des incidents (SNI).

\section{La science de l'amélioration des soins de santé}

Batalden et Davidoff ont défini l'amélioration de la qualité comme « les efforts combinés et constants pour apporter les changements qui entraîneront de meilleurs devenirs pour les patients (la santé), une meilleure performance du système (les soins), et un meilleur développement professionnel (l'apprentissage) ». ${ }^{9}$ Cette définition nécessite la conviction que « tous les intervenants dans 
les soins de santé doivent reconnaître qu'ils ont deux emplois quand ils viennent travailler chaque jour : faire leur travail et l'améliorer. » ${ }^{9}$

Les efforts systémiques destinés à améliorer la qualité, la sécurité et la valeur des soins de santé ont augmenté, et la science de l'amélioration des soins de santé aussi. Les normes SQUIRE 2.0 (Standards for Quality Improvement Reporting Excellence - ou Normes pour l'excellence de communication des améliorations de la qualité) révisées en 2015 mettent l'emphase sur trois composantes clés pour la communication académique des améliorations dans les soins de santé : la théorie formelle et informelle dans la planification, la mise en ouvre et l'évaluation du travail d'amélioration; le contexte dans lequel le travail est effectué; et l'étude de l'intervention. ${ }^{7}$ Les efforts visant à améliorer les soins de santé peuvent être complexes et dépendent de leur contexte, et les Directives SQUIRE 2.0 ont été conçues de manière à pouvoir être appliquées dans plusieurs approches méthodologiques, tels que les cycles Planifier-Faire-Étudier-Agir dans des études à site unique ou randomisées multicentriques. Les Directives SQUIRE 2.0 garantissent une cohérence dans la communication, étant donné que les améliorations se fondent sur l'épistémologie de divers domaines.

\section{Intégration entre amélioration de la qualité, formation en sécurité des patients et soins cliniques}

Des directives récentes de la SCA recommandent d'avoir recours «à une approche systématique pour surveiller la qualité des soins anesthésiques offerts par les membres du département d'anesthésie à la grandeur de l'établissement de soins de santé $» .{ }^{2}$ Une description narrative de Pysyk et coll. souligne la création, la gestion et les expériences d'un comité de qualité et sécurité des patients (QSP) dans un département canadien d'anesthésiologie et de médecine de la douleur. ${ }^{10}$ Bien que la structure et la fonction du comité local de QSP adhèrent aux politiques institutionnelles locales et aux lois provinciales, les auteurs illustrent l'utilisation d'un système rigoureux de notification des incidents et des données de résultats des soins cliniques institutionnels afin d'instaurer des changements et d'éduquer leurs pairs concernant les nouvelles questions de sécurité des patients et de qualité des soins.

L'amélioration de la qualité et la sécurité des patients sont considérées comme des compétences de base pour les professionnels de la santé tels que les anesthésiologistes et les résidents en anesthésiologie. Afin d'améliorer les devenirs pour les patients, leurs familles et les collectivités, le Collège royal des médecins et chirurgiens du Canada a proposé quatre directions stratégiques pour incorporer la formation en amélioration de la qualité et sécurité des patients (AQSP) dans les soins cliniques. Il s'agit de 1) prioriser l'intégration de la formation en AQSP et des soins cliniques; 2) bâtir des structures et mettre en œuvre des processus afin d'intégrer la formation en AQSP et les soins cliniques; 3 ) développer les capacités pour la formation en AQSP à de multiples niveaux; et 4) aligner les résultats éducatifs et les devenirs des patients afin d'améliorer la qualité et la sécurité des patients. ${ }^{11}$

Bien que l'implication des médecins soit importante pour la réussite d'un projet, les anesthésiologistes manquent souvent de formation en amélioration de la qualité, ce qui peut rendre difficile leur participation dans les initiatives d'amélioration des soins de santé. Les comités de qualité et sécurité des patients et les SNI, tels que le Système canadien de rapport des incidents anesthésiques, ${ }^{\mathrm{A}}$ peuvent nous aider à identifier les occasions potentielles d'amélioration au sein des départements d'anesthésiologie, de médecine périopératoire et de prise en charge de la douleur. Silver et coll. ont publié un article qui aidera les professionnels de la santé à se familiariser avec la conception d'un projet d'amélioration des soins de santé. ${ }^{12}$ Le recrutement d'une équipe de base dédiée au changement et possédant une expertise dans les problèmes de qualité des soins, en leadership et en gestion des changements permettra d'identifier les acteurs clés d'un tel projet, y compris les patients. Un modèle d'amélioration, tel que Six Sigma, Lean et le Model for improvement peuvent servir de cadre scientifique pour guider le processus d'amélioration des soins de santé. Il a été démontré que les initiatives d'amélioration de la qualité avaient des impacts positifs sur la sécurité et les soins des patients. ${ }^{13}$ Il a été établi que le soutien au leadership, l'utilisation des données et les 'champions' à l'interne constituaient d'importants facilitateurs en matière d'amélioration de la qualité. Les obstacles à la réussite des projets d'amélioration de la qualité sont le manque de temps et la priorisation de l'amélioration de qualité par rapport aux autres tâches.

\section{Création d'une culture de la sécurité}

L'article de Merchant met en lumière les limites potentielles à l'utilisation des systèmes de gestion de l'information (SGI) disponibles dans le cadre des initiatives d'amélioration de la qualité. Alors que la base de données de la salle d'opération du réseau FHA a été utilisée pour examiner l'observance de l'heure planifiée des cas, le SGI disponible n'a pas permis de relier les incidents de sécurité des patients associés à la non-observance du programme aux directives de jeûne ou aux modifications apportées au programme opératoire afin de respecter les directives de jeûne. Les SGI devraient être conçus de 
manière à offrir des données interrogeables et à pouvoir être utilisés pour communiquer avec les autres bases de données de l'institution lorsque possible. ${ }^{2}$

Les anesthésiologistes et autres fournisseurs de soins de santé ont l'objectif commun de reconnaître les incidents de sécurité des patients, d'en identifier les causes et d'apporter des changements à l'échelle du système afin de réduire le risque médical. L'objectif du Système canadien de rapport des incidents anesthésiques (SCRIA) est de colliger tous les incidents, erreurs et complications survenant en période périopératoire. ${ }^{14}$ Nous sommes intimement convaincus que les devenirs périopératoires peuvent être améliorés en étudiant et en comprenant les éléments qui contribuent aux incidents de sécurité des patients, tant au niveau local que national. Un cadre intégré de SGI pour la gestion de la sécurité, de la qualité et du risque, tels que le SCRIA, est nécessaire.

\section{Conclusion}

La collecte de données seule ne suffit pas au changement. ${ }^{15}$ La mise en œuvre réussie des mesures d'amélioration aux niveaux local et national favorisera la création d'un cycle de qualité continu et contribuera à l'amélioration de la sécurité des patients en période périopératoire. Cela ne sera possible que s'il y a un engagement envers une « culture de la sécurité » et un système de soins périopératoires canadien qui place l'excellence dans les soins et la sécurité des patients comme priorité numéro un.

\section{Conflicts of interest None.}

Funding statement None.

Editorial responsibility This submission was handled by Dr. Philip M. Jones, Associate Editor, Canadian Journal of Anesthesia.

\section{Conflit d'intérêt Aucun}

Déclaration de financement Aucune.

Responsabilité éditoriale Cet article a été traité par Dr Philip M. Jones, rédacteur adjoint, Journal canadien d'anesthésie.

\section{References}

1. Merchant $R N$. Slated versus actual operating room entry time in a British Columbia health authority. Can J Anesth 2020; 67: DOI: https://doi.org/10.1007/s12630-020-01604-9.

2. Dobson G, Chow L, Filteau L, et al. Guidelines to the practice of anesthesia - revised edition 2020. Can J Anesth 2020; 67: 64-99.

3. Cook TM, Woodall N, Frerk C; Fourth National Audit Project. Major complications of airway management in the UK: results of the Fourth National Audit Project of the Royal College of Anaesthetists and the Difficult Airway Society. Part 1: anaesthesia. Br J Anaesth 2011; 106: 617-31.

4. Perlas A, Van de Putte P, Van Houwe P, Chan VW. I-AIM framework for point-of-care gastric ultrasound. $\mathrm{Br} \mathrm{J}$ Anaesth 2016; 116: 7-11.

5. De Jonghe B, Fajardy A, Mérian-Brosse L, et al. Reducing preoperative fasting while preserving operating room scheduling flexibility: feasibility and impact on patient discomfort. Acta Anaesthesiol Scand 2016; 60: 1222-9.

6. James Lind Alliance. Priority Setting Partnership. Anesthesia (Canada) Top 10. https://doi.org/10.1007/s12630-020-01607-6. Available from URL: http://www.jla.nihr.ac.uk/priority-settingpartnerships/anaesthesia-canada/top-10-priorities.htm Accessed February 2020.

7. Ogrinc G, Davies L, Goodman D, Batalden P, Davidoff F, Stevens D. SQUIRE 2.0 (Standards for QUality Improvement Reporting Excellence): revised publication guidelines from a detailed consensus process. Am J Med Qual 2015; 30: 543-9.

8. Brown B, Khemani E, Lin C, Armstrong K. Improving patient flow in a regional anaesthesia block room. BMJ Open Qual 2019; . https://doi.org/10.1136/bmjoq-2018-000346.

9. Batalden PB, Davidoff $F$. What is "quality improvement" and how can it transform healthcare? Qual Saf Health Care 2007; 16: 2-3.

10. Pysyk CL, Filteau L, Baxter A. Quality and patient safety committee structure and activities in an academic department of anesthesiology: a narrative description. Can J Anesth 2020; 67: $100-8$.

11. Wong BM, Baum KD, Headrick LA, et al. Building the bridge to quality: an urgent call to integrate quality improvement and patient safety education with clinical care. Acad Med 2020; 95: 59-68.

12. Silver SA, Harel Z, McQuillan R, et al. How to begin a quality improvement project. Clin J Am Soc Nephrol 2016; 11: 893-900.

13. Zoutman DE, Ford BD. Quality improvement in hospitals: barriers and facilitators. Int J Health Care Qual Assur 2017; 30: 16-24.

14. Beattie WS, Culwick MD, Grocott HP. Canadian Anesthesia Incident Reporting System (CAIRS): the Canadian Anesthesiologists' Society's national patient safety initiative. Can J Anesth 2018; 65: 749-56.

15. Merry AF. Safety in anaesthesia: reporting incidents and learning from them. Anaesthesia 2008; 63: 337-9.

Publisher's Note Springer Nature remains neutral with regard to jurisdictional claims in published maps and institutional affiliations. 